61.3 in the successful endoscopy group $(P=0.077)$. Both groups had an equal number of male and female patients $(\mathrm{P}=1.000)$.

Predictors of failure of endoscopy include initial presentation with haematemesis, initial systolic blood pressure of $\leq 90 \mathrm{mmHg}$, initial heart rate of $\geq 95$ per minute, serum urea $\geq 13 \mathrm{mmol} / \mathrm{L}$, Forrest Ia or $\mathrm{Ib}$ ulcer at first endoscopy and Rockall score of $\geq 5$. (table 1 )

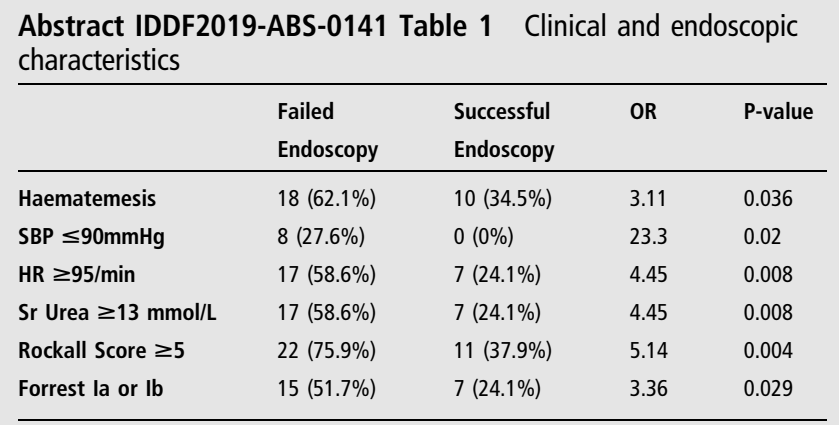

SBP: systolic blood pressure, HR: heart rate, OR: odds ratio

Conclusions It is crucial to identify predictors of failure of endoscopic therapy in bleeding peptic ulcers in order to institute aggressive therapeutic options including early surgical intervention or angioembolization to reduce associated morbidity and mortality.

\section{IDDF2019-ABS-0143 ASSOCIATION BETWEEN BARIATRIC SURGERY AND MACROVASCULAR DISEASE OUTCOMES IN PATIENTS WITH TYPE 2 DIABETES AND SEVERE OBESITY: A META- ANALYSIS OF COHORT STUDIES}

${ }^{1}$ Salman Hussain*, ${ }^{2}$ Ali Nasir Siddiqui, ${ }^{3}$ Abul Kalam Najmi. 'Department of Pharmaceutical Medicine, School of Pharmaceutical Education and Research, Jamia Hamdard, India; ${ }^{2}$ Publication writer, GCE Solutions Inc. Jasola DLF Tower B, New Delhi, India; ${ }^{3}$ Department of Pharmacology, School of Pharmaceutical Education and Research, Jamia Hamdard, India

\subsection{6/gutjnl-2019-IDDFabstracts.164}

Background Severely obese Type 2 Diabetes Mellitus (T2DM) patients are on increased risk of mortality, morbidity and macrovascular complications. Real-world evidence suggested a reduced rate of macrovascular complications following bariatric surgery. So, we undertook this meta-analysis to understand the impact of bariatric surgery in macrovascular disease outcomes in severely obese T2DM patients.

Methods A comprehensive search was performed in PubMed, and Embase database from inception to October 2018. The inclusion criteria were as follows: (a) obese T2DM patients (BMI $>35 \mathrm{~kg} . / \mathrm{m}^{2}$ ) who underwent bariatric surgery (b) provided hazard ratio (HR) or relative risk (RR). Study quality was assessed using the Newcastle-Ottawa Scale. The primary outcome was to assess the impact of bariatric surgery and macrovascular complications risk. Statistical analysis was performed using Review Manager software.

Results This meta-analysis comprised of five studies with a total of 49211 participants (75\% female) of which 14434 underwent bariatric surgery and 34777 underwent usual care. The participants in the bariatric surgery group had a mean age of $48 \pm 8.98$ years, mean BMI of $44.67 \pm 6.3 \mathrm{~kg} / \mathrm{m}^{2}$ and mean diabetes duration and a follow-up period of $5.48 \pm$ 5.11 years and 10.96 years, respectively. Included studies were of high quality.

Participants who underwent bariatric surgery group had significantly lower risk of macrovascular complications as compared to participants who underwent nonsurgery group with a RR of 0.50 (95\% CI: $0.35-0.73$ ), $p=0.0003$ ) (figure 1). Subgroup analysis revealed studies conducted in US showed higher reduction $[\mathrm{RR} \quad 0.41 \quad(95 \%$ CI: $0.32-0.53, \mathrm{p}=$ $<0.00001)]$ in incident macrovascular complications as compared to those conducted in other parts of the world [RR 0.71 (95\% CI: $0.56-0.89), p=0.003]$. The risk of all-cause mortality was also significantly lower in bariatric surgery group (RR of 0.39 [95\% CI: $0.30-0.50$ ], p $=<0.00001$ ).

Conclusions Our meta-analysis supports the benefit of bariatric surgery in reducing macrovascular complications in morbidly obese T2DM patients. However, the observational design of included studies might have precluded the inference despite adjustment of confounding factors. Hence, these findings need to be confirmed in well-designed randomized trials.

\section{IDDF2019-ABS-0144 EFFICACY OF 7-DAY VONOPRAZAN AND AMOXICILLIN DUAL THERAPY AS FIRST- LINE HELICOBACTER PYLORI TREATMENT: PROTOCOL OF MULTI-CENTER, NON- INFERIORITY, RANDOMIZED CONTROL TRIAL}

${ }^{1}$ Sho Suzuki*, 'Takuji Gotoda, ${ }^{2}$ Hitoshi Shibuya, ${ }^{1}$ Chika Kusano, ${ }^{1}$ Hisatomo Ikehara, ${ }^{1}$ Ryoji Ichijima, ${ }^{3}$ Koichi Kawabe, ${ }^{3}$ Toyotaka Kasai, ${ }^{3}$ Hiroyuki Eto, ${ }^{4}$ Yoshioki Yoda, ${ }^{4}$ Masahiko Ohtaka, ${ }^{5}$ Motoki Ohyauchi, ${ }^{5}$ Hirotaka Ito, ${ }^{6}$ Masashi Kawamura, ${ }^{6}$ Yohei Ogata, ${ }^{7}$ Moriyasu Nakahara. ${ }^{1}$ Nihon University School of Medicine, Japan; ${ }^{2}$ Yuri Kumiai General Hospital, Japan; ${ }^{3}$ Fukaya Red Cross Hospital, Japan; ${ }^{4}$ Yamanashi Koseiren Health Care Center, Japan; ${ }^{5}$ Osaki Citizen Hospital, Japan; ${ }^{6}$ Sendai City Hospital, Japan; ${ }^{7}$ Chichibu Municipal Hospital, Japan

\subsection{6/gutjnl-2019-IDDFabstracts. 165}

Background Recent international guidelines recommend fourdrug combination therapies as first-line treatment to overcome Helicobacter pylori antibiotic resistance and achieve sufficient

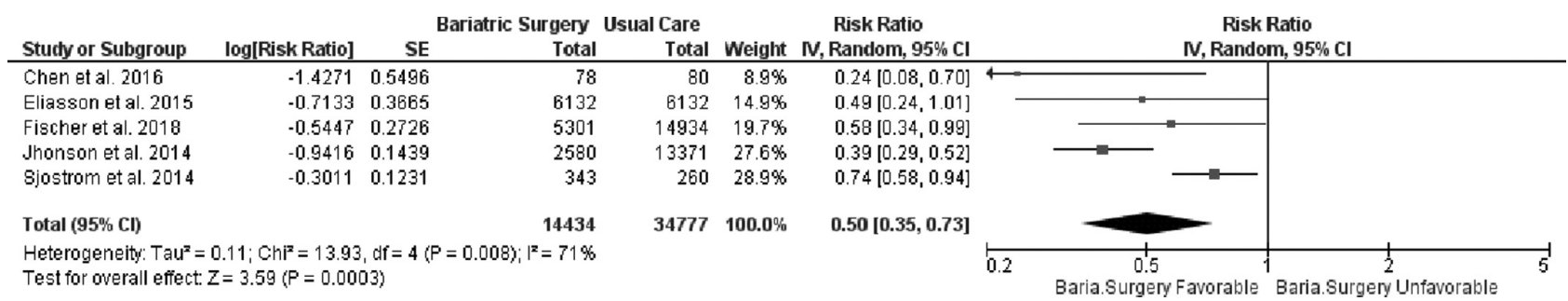

Abstract IDDF2019-ABS-0143 Figure 1 Forest plot showing impact of bariatric surgery in reducing macrovascular complications 